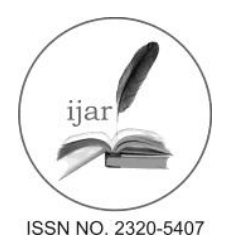

Journal homepage: http://www.journalijar.com

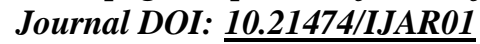

RESEARCH ARTICLE

ANALYSIS OF LOCATION AWARE PROTOCOLS IN WIRELESS SENSOR NETWORKS.

\title{
Manoj Kumar.
}

Assistant Professor, BBK DAV College for Women, Amritsar-India -143001

\section{Manuscript Info}

\section{Manuscript History:}

Received: 16 March 2016

Final Accepted: 22 April 2016

Published Online: May 2016

Key words:

WSN, energy efficiency, location

aware routing, GPSR, GEAR, GAF

*Corresponding Author

\begin{abstract}
Location based routing is an upcoming research domain due to their ability to improve energy efficiency of a wireless sensor network; energy efficiency is an important factor deciding the performance of a WSN. Location aware routing protocols utilize location information to discover and maintain a route, thus selecting the best route to minimize the energy consumption. The research paper presents an analysis of mainly used location aware routing protocols Greedy Perimeter Stateless Routing (GPSR), Geographic and Energy Aware Routing (GEAR) and Geographic Adaptive Fidelity (GAF).
\end{abstract}

\section{Manoj Kumar.}

Copy Right, IJAR, 2016,. All rights reserved.

\section{Introduction:-}

Wireless sensor network technology has become an interesting and attractive research area for industry and academia. Typical a wireless sensor network consists of large number of small size, low cost and low power sensors. Each sensor has a sensing, processing and transmission unit. The sensor nodes communicate and collaborate with each others to fulfill a task. Components of a sensor node are shown in fig.1 [9].

Routing is one of the critical technologies in computer networks and routing in WSNs is more challenging as a result of their various inherent characteristics [10].Firstly due to constrained resources in terms of power, processing and transmission bandwidth. Secondly, it is challenging to design a global addressing scheme as Internet Protocol (IP), as IP cannot be applied to WSNs as address updating in a dynamic WSN can cause heavy overhead. Thirdly, data collection by many sensor nodes usually results in a high probability of data redundancy, which must be considered by routing protocols. Fourthly, most applications of WSNs require the only communication scheme of many-to-one rather than multicast or peer to peer. In time-critical applications of WSNs, data transmissions should be done within a certain time period [11].

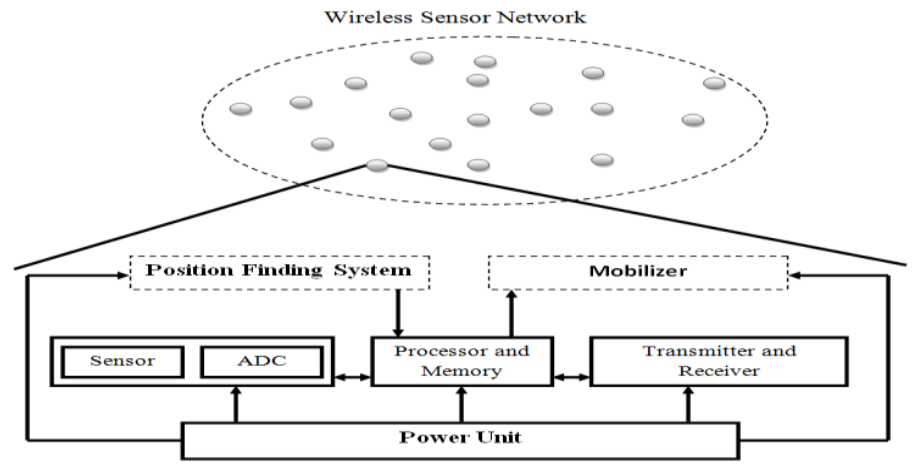

Fig 1: WSN components 


\section{WSN structure:-}

WSN can be based of two types of models

* Unstructured Model:-

Sensor nodes are randomly deployed and can have uncovered regions. It is left unattended and hence maintenance is difficult.

* Structured Model:-

Sensor nodes are fever and deployed in preplanned manner. There is no uncovered region and maintenance cost is less

\section{Sensor network designs objectives:-}

A WSN should take appropriate action according a situation, able to process and observe physical world quantities. Keeping these capabilities in view sensor network should have following designs objectives:

1. Reliability: Routing protocols for sensor network must provide reliability by ensuring reliable delivery of data. Reliability become more important in case of noisy and error prone communication channels.

2. Security: Sensor network must be secure against unauthorized access or attack.

3. Scalability: Routing protocols should be scalable to different network sizes as sensor network can be very large or small.

4. Low power consumption: Sensor nodes should power efficient i.e. low power consuming nodes are required.

5. Efficient use of bandwidth: In order to have efficient use of channel there should be proper use of bandwidth.

6. Fault tolerant: Mostly sensor network are deployed in adverse condition so they can be subjected to failure .So in order to have recovery from failure sensor network should be fault tolerant.

7. Cost of sensor node: The cost of single sensor node should be low to reduce the cost of sensor network.

\section{Routing Protocols in WSN:-}

WSN routing protocols can be categorized as under

* Flat.

* Hierarchical.

* Location based.

Flat Routing Protocols:-

In flat network, each node typically plays the same role and sensor nodes work together to perform the sensing. Due to the large number of such nodes, it is not feasible to assign a global identifier to each sensor node. This consideration has led to data centric routing protocols, where the base station (BS) sends queries to certain sensing regions and waits for data from the sensors located in the those regions. Since data is being requested through queries, attribute-based naming is necessary to specify the properties of data. Before the use of data centric routing protocols, e.g., SPIN and Directed Diffusion, were utilize to minimize energy consumption with the help of data negotiation and elimination of redundant paths.

\section{Hierarchical Protocols:-}

Hierarchical routing is mainly two-layer routing where one layer is used to select cluster heads and the other layer is used for routing In a hierarchical protocols, low energy nodes can be used to perform the sensing of the target and higher energy nodes can be used to process and send the information. LEACH, PEGASIS, TEEN APTEEN are an efficient way to lower energy consumption within a cluster and by performing data aggregation and fusion in order to decrease the number of transmitted messages to the BS.

\section{Location Based Routing Protocols:-}

In location based routing, sensor nodes are identified using their locations. The incoming signal strength is used to find the distance between neighboring nodes. Relative coordinates of neighboring sensor nodes can be obtained by exchanging this information between neighboring nodes [1]. If nodes are equipped with a GPS (global positioning system) receiver, the location of nodes can be calculated by communicating with a satellite, using GPS receiver .In order to minimize the use of energy, location based routing demand that sensor nodes should go to sleep mode in case there is no activity. More energy savings can be obtained by having as many sleeping nodes in the network as possible [2]. 


\section{Location based protocols:-}

Geographic and Energy Aware Routing (GEAR):-

Geographic and Energy Aware Routing (GEAR) is location based routing protocol that uses energy aware and geographically-informed scheme to route a packet only towards the destination area instead routing a packet towards the whole network[5].Using restricted forwarding GEAR can reduce the energy consumption to improve the network life time. The GEAR has two main phases.

Phase 1: Routing packets towards the target area (region): when a node receives a packet, it checks its neighbors list to select the nodes which closer to the destination than itself. If there is more than one node, the nearest neighbor to the destination is selected as the next hop and if neighboring node is further than the node itself, this means there is a hole. In such a case, one of the neighbors is selected to forward the packet based on the learning cost function. Each node $\mathrm{N}$ maintains state $\mathrm{h}(\mathrm{N}, \mathrm{R})$ which is called learned cost to the target region $\mathrm{R}$.

* Each node infrequently updates neighbor of its cost.

* When a node wants to forward a packet, it checks the learned cost to that target region of all its neighbors.

* If a node does not have the learned cost of a neighbor to a region, the estimated cost is computed as follows:

$$
c\left(N_{i}, R\right)=\alpha d\left(N_{i}, R\right)+(1-\alpha) e\left(N_{i}\right)----(1)
$$

In equation $1, \alpha=$ tunable weight ranging from 0 to $1, \mathrm{~d}\left(\mathrm{~N}_{\mathrm{i}}, \mathrm{R}\right)=$ normalized the largest distance among neighbors of $\mathrm{N}$ and $\mathrm{e}\left(\mathrm{N}_{\mathrm{i}}\right)=$ normalized the largest consumed energy among neighbors of $\mathrm{N}[5]$.

Phase 2: Forwarding the packets within the interested region: If the packet has reached the region, it can be diffused in that region by either recursive geographic forwarding or restricted flooding. Restricted flooding is good when the sensors are not densely deployed.

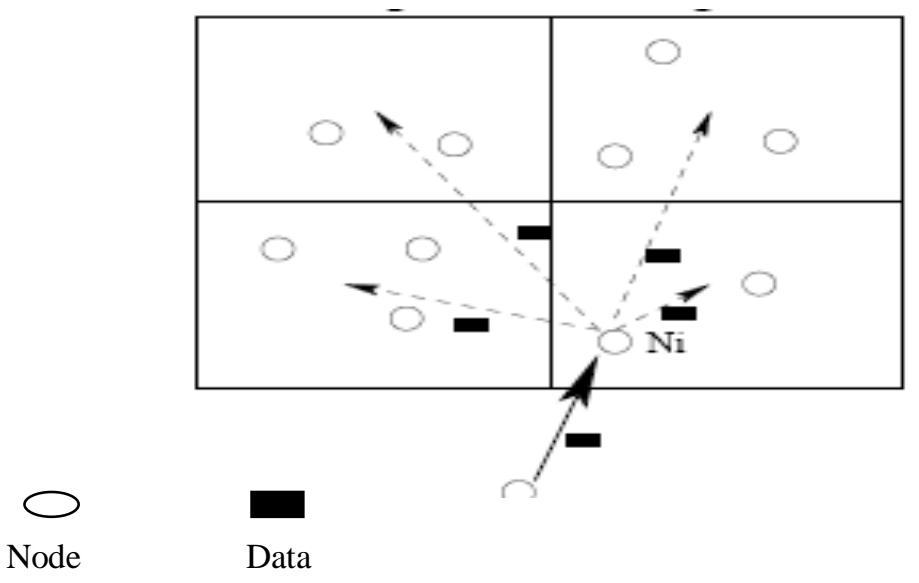

Fig 2: Recursive geographic forwarding in GEAR

\section{Greedy Perimeter Stateless Routing (GPSR):-}

Greedy Perimeter State Routing (GPSR) is a responsive and efficient routing protocol for wireless networks [7][8]. Unlike traditional routing algorithms which use shortest paths and transitive reach ability to determine routes, GPSR uses the correspondence between geographic position and connectivity in a wireless network, by using the location of nodes to make packet forwarding decisions. It proposes the aggressive use of geography to achieve scalability. Although the GPSR approach reduces the number of states a node should keep, it has been designed for general mobile ad hoc networks and requires a location service to map locations and node identifiers. The GPSR uses term greedy as it is based on forwarding decision which is sending packet from one node to another. The GPSR uses two forwarding methods greedy forwarding and perimeter forwarding .Perimeter forwarding is used when greedy forwarding cannot be utilized.

\section{Greedy forwarding:-}


Greegy forwarding can be used if each node knows its radio neighbors .In greedy forwarding a packet is forwarded to a neighboring node which is closet to the destination in next hop.e.g as shown in fig. 3 , as a packet arrives at $\mathrm{x}$ node, it forward the packet to $\mathrm{y}$ which is closer to destination D in next hop. In GPSR packets are marked by their source with their destinations' locations and forwarding node make greedy choice in selecting packet next hop. Each node maintain local table in which locations of its radio neighbors are stored. Whenever a packet arrives at a node it checks its local table to find a node which is nearest to the destination node and forward packet to that corresponding node [12].

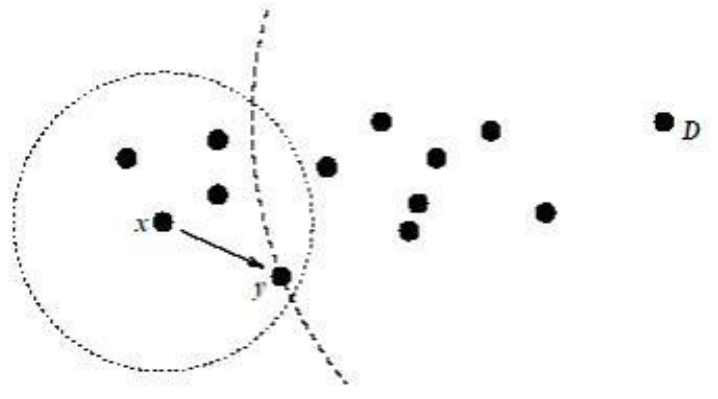

Fig 3: Greedy forwarding

Greedy forwarding is a recursive process in which forwarding packet to the nearest neighbor of the destination continue until it reaches destination. Each node uses a simple beacon algorithm to find the locations of its neighbors. Each node sends a beacon containing its IP address and location, if a beacon from a neighbor is not received for period of time greater than time out interval, a GPSR router assumes that neighbor either gone out of range or failed and delete the neighbor from its local table [13]. Greedy forwarding rely only on the information of the forwarding nodes immediate neighbors. The state required is negligible and independent of total number of destinations in the network it depend only on the density of the sensor nodes

\section{Perimeter forwarding:-}

It is used when there is void between the node where packet arrived and the destination i.e. there no node between them .In that case packet is forwarded to the destination using perimeter of void .This is called a right hand rule.

As shown in fig. 4 nodes $x$ is nearer to $D$ than $w$ and $y$, there are two possible paths $x->y->z->D$ and $x->w->v->D$, node $\mathrm{x}$ does not select $\mathrm{y}$ or node $\mathrm{w}$ to forward the packet rather considering the location of destination $\mathrm{D}, \mathrm{x}$ lies on local maximum[13][14]. Greedy forwarding will not be used. The right hand rule is used to find out a possible path around a void to the destination node $\mathrm{D}$ when packet arrive at node $\mathrm{x}$ from $\mathrm{y}$ next edge travel will be the next one sequentially counter clockwise about $\mathrm{x}$ from edge $(\mathrm{x}, \mathrm{y})$. The right-hand rule traverses the interior of a closed polygonal region in clockwise order. As shown in fig 4 edges are traversed in the order $y \rightarrow x \rightarrow z->y[13][14]$.

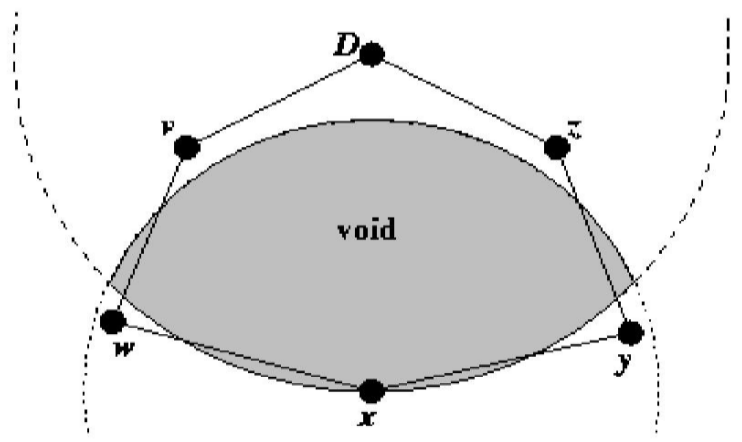

Fig 4: Void between node $\mathrm{x}$ and destination $\mathrm{D}[14]$

\section{Geographic adaptive fidelity (GAF):-}

Geographic adaptive fidelity is another commonly used location based routing protocol. It was basically designed for MANETs but can be used in WSNs. The sensing area is divided into fixed zones to form a virtual grid. Each 
node utilizes its GPS receiver to find its location to associate itself with a particular point in a virtual grid. All the nodes associated with same point considered equivalent. Nodes in GAF can be in one of three states sleeping, discovery and active. Nodes start in discovery state with their radio receiver on to find other nodes within virtual grid. Nodes are in active state when they are participating in routing and sleep state indicate state when a node turn off its radio.GAF minimize energy by turning off unnecessary nodes e.g. nodes will elect one sensor node to stay awake for a period of time and they enter into sleep state to conserve their energy.

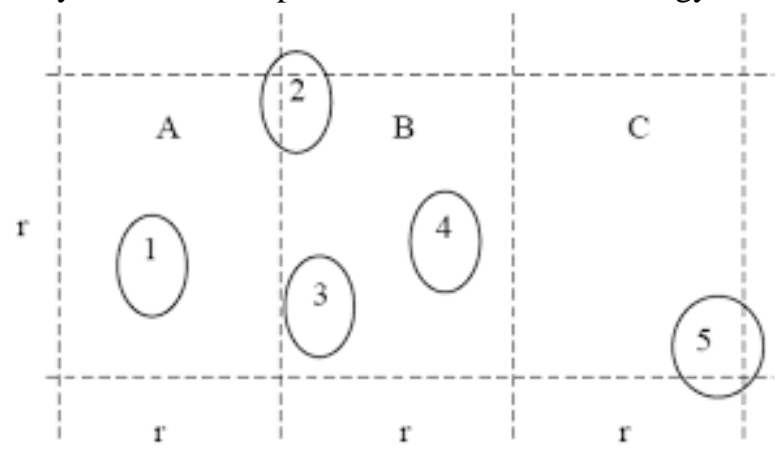

Fig 5: Virtual grid in GAF.

Virtual grid is shown in fig. 5, Node 1 can reach any of node 2, 3 and 4 so 2, 3 and 4 are equivalent .Any two nodes out of 2,3 and 4 can go sleep state without affecting routing. Each node estimates its time of leaving the grid and sends it to its neighbors.

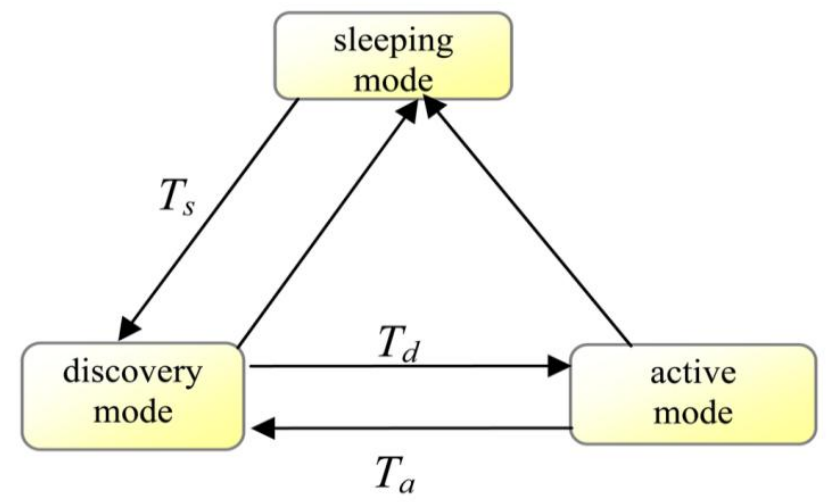

Fig 6: State transition of GAF [3].

Nodes exchange node Id, grid Id and various timers. Timer Td tells when a node sends discovery message and enter into active state, timer Ta determine when a node goes back to discovery state and timer Ts determine when a node wake up to enter into discovery state.GAF aims to maximize the network lifetime by reaching a state where each grid has only one active sensor having highest residual energy. A sensor in the active state has higher rank i.e. longer lifetime then a sensor node in the discovery state.

\section{Comparison of location based protocols:-}

Comparison of location based protocols is given in table 1. Location based protocols are compared using data delivery model, scalability, overhead, power management and mobility. GAF provide high scalability but also high overhead. GEAR has low scalability but also low overhead. Three GAF, GEAR and GSPR offer limited mobility and none of these uses Qos as a metric for routing.

Table 1. Comparison of Location Based Protocols.

\begin{tabular}{|l|l|l|l|l|l|l|}
\hline Routing Protocols & Data delivery model & Scalability & Power management & Overhead & Qos & Mobility \\
\hline GEAR & Demand based & Average & Average & High & No & Limited \\
\hline GPSR & $\begin{array}{l}\text { Greedy or perimeter } \\
\text { forwarding }\end{array}$ & Medium & Average & High & No & Limited \\
\hline GAF & Virtual grid & High & Average & High & No & Limited \\
\hline
\end{tabular}




\section{Conclusion:-}

Routing in wireless sensor networks present all together different challenges as compared to routing in wired networks. One of the considerable design challenges is energy efficient routing in energy constrained WSNs. Location based protocols present a solution to improve the energy efficiency of a WSN. GEAR, GPSR and GAF are commonly used location based protocols.GAF is highly scalable but offer more overhead and provide limited mobility whereas GEAR offers demand based delivery model but has average scalability. Each of location based protocol has its own shortcomings so the use of a location based protocol depends upon the application and environment in which WSN is deployed.

\section{References:-}

1. N. Bulusu, J. Heidemann, D. Estrin "GPS-less low cost outdoor localization for very small devices", Technical report 00- 729, Computer science department, University of Southern California, Apr. 2000.

2. Jamal N. Al-Karaki Ahmed E. Kamal "Routing Techniques in Wireless Sensor Networks: A Survey" supported in part by the ICUBE initiative of Iowa State University, Ames, IA 50011.

3. Le Xuan Hung, Ngo Trong Canh, Sungyoung Lee, Young-Koo Lee and Heejo Lee "An Energy-Efficient Secure Routing and Key Management Scheme for Mobile Sinks in Wireless Sensor Networks Using Deployment" Sensors , 8(12), 7753- 7785,2008.

4. Y. Xu, J. Heidemann, and D. Estrin, "Geography-informed energy conservation for ad hoc routing", in Proceedings of the 7th Annual ACM/IEEE International Conference on Mobile Computing and Networking (MobiCom_01), Rome, Italy, Jul. 2001.

5. Nirupama Bulusu, John Heidemann, and Deborah Estrin. "Gps-less low cost outdoor localization for very small devices". IEEE Personal Communications Magazine, 7(5), 28-34, October 2000.

6. Y. Yu, D. Estrin, and R. Govindan, "Geographical and Energy-Aware Routing: A Recursive Data Dissemination Protocol or Wireless Sensor Networks", UCLA Computer Science Department Technical Report, UCLA-CSD TR-01-0023, 2001

7. Varun G Menon,Jogi Priya P M,Joe Prathap P M "Analyzing the Behavior and Performance of Greedy Perimeter StatelessRouting Protocol in Highly Dynamic Mobile Ad Hoc Networks" Life Science Journal, 10(2), 2013.

8. M. Mauve, J. Widmer and H. Hartenstein "A Survey on Position Based Routing in Mobile Adhoc Networks" IEEE network Magazine, 15(6), 2001.

9. Shio Kumar Singh, M P Singh and D K Singh "A Survey of Energy-Efficient Hierarchical Cluster-Based Routing in wireless Sensor Network," Int J. of Advanced Networking and Applications 2(2), 570-580, 2010.

10. Sustainable Wireless Sensor Networks; Seah, W Tan, Y. Eds.; InTech Open Access Publisher: Rijeka, Croatia, 2010.

11. Xuxun Liu “ A Survey on Clustering Routing Protocols in Wireless Sensor Networks " Sensors, 11113- 11153 ,2012.

12. Zayene M A, Tabbane N, Elidoudi R, "Performance Evaluation of Greedy Perimeter Stateless Routing Protocol in Ad HocNetworks" Fourth IEEE International Conference on Computer Sciences and Convergence Information Technology, 2009.

13. Varun G Menon, Jogi Priya P M, Joe Prathap P M "Analyzing the Behavior and Performance of Greedy PerimeterStateless Routing Protocol in Highly Dynamic Mobile Ad Hoc Networks" Life Science Journal, 10(2), 2013.

14. Brad Karp, H. T. Kung, "GPSR: Greedy Perimeter Stateless Routing for Wireless Networks", ACM International Conference on Mobile Computing and Networking, MobiCom, 2000. 